\title{
The roles of data analytics in the fashion industry
}

\begin{abstract}
The importance of data has been gradually acknowledged by fashion professionals to improve sales and margins because fashion brands and retailers need to develop, manufacture, and sell styles that resonate with consumers. Lately, advancements in data analytics, machine learning, and computing power, the value of utilizing artificial intelligence (AI)-based software or applications has been well acknowledged by fashion brans and retailers who want to apply a data-driven decision-making approach to develop more efficient fashion design, merchandising, and marketing strategies. In this research, it is intended to review the roles and importance of data analytics in the fashion merchandising process. AI-powered data analytics applications or services that are currently available in the fashion industry are also introduced. Finally, a need for developing courses or programs focusing on fashionspecific data analytics in higher education is addressed as more and more fashion brands and retailers are trying to hire fashion data analysts. Collaboration with technology partners who are providing AI-powered data analytics services to fashion brands and retailers is needed to educate fashion students with practical knowledge and skills.
\end{abstract}

Volume 6 Issue 3 - 2020

\section{Keunyoung Oh}

Department of Fashion and Textile Technology, State University of New York College at Buffalo State, USA

\begin{abstract}
Correspondence: Dr keunyoung Oh, Department of Fashion and Textile Technology, State University of New York College at Buffalo State, 1300 Elmwood Ave, Technology Building 306, USA, Email ohk@buffalostate.edu
\end{abstract}

Received: May 29, 2020 | Published: June 25, 2020

\section{The roles of data analytics in the fashion industry}

Data is abundant in the current fashion business environment as sales data, product information, and consumer data are constantly collected and analyzed. The importance of data has been gradually acknowledged by fashion professionals to improve sales and margins because fashion brands and retailers need to develop, manufacture, and sell styles that resonate with consumers. Lately, with advancements in data analytics and machine learning, fashion brands and retailers have become more aware of the value of utilizing Artificial Intelligence (AI)-based software or applications to create efficient fashion design, merchandising, and marketing strategies. While many retailers like Amazon or pure online players have been aggressively finding ways to apply advanced data analytics to improve performance in design and product development, merchandising, marketing, operations, channel management, and human resources, traditional fashion brands and retails tend to rely on experts' gut instinct rather than data-driven decision making using advanced data analytics. ${ }^{1}$ However, a growing interest and investment in embracing big data and data analytics has been observed in the fashion industry. ${ }^{2}$ The most significant reason fashion brands and retailers are seeking the ways to optimize data analytics is to improve their product offerings through precise personalization for targeted customers, which would eventually bring higher sell-through and profitability. According to the survey done by JDA Software Inc. in 2018, $43 \%$ of fashion brands and retailers planned to invest in customer-based data science in the next five years for converting customer data into personalized merchandising assortments based on their lifestyle and localized trends. ${ }^{3}$ Ecommerce retailers as well as brick-and-mortar stores in the fashion industry are now in need of incorporating data analytics and AI technologies in their design and merchandising processes. In this review, the roles and importance of data analytics in the fashion industry especially in the fashion merchandising process are explored. As data analytics, machine learning, and AI-based applications are often mentioned together and used interchangeably, AI-based merchandising applications are reviewed. It is also discussed how to improve students' data literacy in the fashion-related programs in higher education.

\section{The roles and importance of data analytics}

Data analytics begins with descriptive analytics focusing on summarizing data in a meaningful and descriptive way to explain what happened in the market. Big data analytics proceeds with advanced, predictive analytics including classical statistics as well as machine learning such as neural networks, natural language processing, sentiment analysis, and more advanced analytics to provide new insight from data and to generate recommendations for possible scenarios. ${ }^{4}$ The availability of machine learning algorithms, big data, and cheap but high-powered computing has brought significant changes in many industries including fashion by providing meaningful insights from various data sources. It has become significant in the mid 2010s to monetarize data by using big data analytics for strategic and operational decision making in the fashion industry. ${ }^{5}$ In the earlier days, data analytics was valued more by ecommerce because their businesses are run on a digital platform and already set up effectively to collect data on consumer behavior online. It is recent that fashion brands and retailers started making use of their internal data to increase sales and profitability and to remain competitive in the market.

Companies' first challenge in collecting and analyzing internal data is data silos that is isolation of data created by different departments or units within a company and without data integration, data cannot be used effectively. Once data from various sources are integrated and data cleaning is performed, the right data metrics that quantifiable and closely related to the companies' objectives should be identified. ${ }^{6}$ Data analytics can even change a top-down segmentation approach to a bottom-up segmentation in the fashion industry. Gruzbarg 7 proposed the four dimensions to make "behavioral and contextual targeting" possible by using data analytics as follows:

a. Demographics like gender, age, or income to understand who the customer is

b. Historic/behavioral data including purchase transactions, cart abandoned, etc. to understand what the customer did in the past and can be used to predict their future behavior

c. Streaming/contextual data such as a customer's current digital behaviors, web pages they are viewing, emails they just open, 
ads they are clicking on to know in what product the customer is interested and whether $\mathrm{s} /$ he is currently in shopping mode

d. Predictive analytics using AI-powered software to predict how the customer is likely to respond in the future by identifying invisible patterns in the previous three dimensions

Using a data-driven segmentation approach will help fashion brands and retailers develop more personalized products and marketing strategies. It is very clear that fashion brands and retailers have become aware of the importance of data analytics in their business decision-making. More efforts need to be made to effectively use the data internally available within the company. In addition, applying data analytics to solve business problems can be extended to working with technology partners who are providing various AIbased applications.

\section{Al-based merchandising applications}

Artificial intelligence (AI) is a combination of technologies including natural language processing, computer visions, machine learning and deep learning algorithms, VR/AR/MR technologies, and more. AI-based merchandising applications can be applied in the data analytics process to provide better answers to business questions generated by fashion brands and retailers. They have outsourced data analytics processes with AI-powered tech companies such as Content square (https://contentsquare.com/), Dynamic Action (https://www. dynamicaction.com/), Oracle Analytics Cloud, IBM Data Analytics, SAP for Retail, etc. Companies providing AI-powered data analytics services to fashion brands and retailers use machine learning algorithms - the branch of artificial intelligence to create executable solutions for how to ensure maximum sales by optimizing product assortment, efficient allocation and a great customer experience. ${ }^{8}$

Fit Analytics, the sizing platform, added a new feature called "Fit Connect" which enables fashion brands and retailers to present a personalized product listing page based on a shopper's size and preferences and product availability. ${ }^{9}$ This approach combining sizing and style intelligence would improve consumers' shopping experience and increase conversion rate and sales after all. Sixty-nine percent of senior executives from national or large regional retailers in the United States indicated that using AI-driven merchandising applications is important to improve merchandising performance; while only $31 \%$ retailers answered that AI-driven merchandising applications will be minimally or completely unimportant. ${ }^{10}$ Skypad software used by $72 \%$ of global luxury brands collects data from various retailers and allows brands to see how their products are performing based on a variety of attributes such as color, size, fabric and geography. It also tracks sales performance via wholesale business and the brands' own stores and online channels. With predictive analytics, they provide insights about season management, what sells, what doesn't sell in different regions, merchandising planning on the retail flor. ${ }^{11}$ Traditional merchandising functions can be improved by utilizing data provided by AI-based merchandising software. However, it does not mean that merchandisers can be replaced by AI-based applications or data analysts. Fashion professionals and data analysts should know each other's language to solve business problems. Fashion professionals would have more information by utilizing AI-powered data analytics and can apply a data-driven decision-making approach more effectively.

Chico's Inc. hired a product pricing and predictive analytics platform provider to improve design, buying, and pricing decisions on their products for their physical stores and e-commerce..$^{12}$ Applying advanced data analytics into the product development or merchandising process can lead fashion companies to increase sell-through and to reduce markdowns by bringing products that consumers want. Custora, a customer analytics platform for apparel brands and retailers uses AIdriven customer insights to grow revenue and increase the customer lifetime value by helping apparel brands and retailers deepen their customer analytics capabilities. ${ }^{13}$ TechStyle Fashion Group has inhouse data science team of which functions include analyzing various customer behaviors across channels as well as purchasing trends and feedback, identifying gaps in their product offerings, and customizing product recommendations and personalization for their customers. ${ }^{14}$ Fashion consumers are increasingly demanding more personalized product offerings and shopping experience especially both online and in physical stores. Fashion brands and retailers need to invest more in AI-powered data analytics to create personalized services to their customers. It would be a great advantage for fashion professionals to become data literate so they can work fluently with data analysts if they cannot perform data analysis or operate AI-based applications.

\section{A growing need for fashion-specific data analytics program}

An increasing demand for data-driven insights and AI-based applications in the fashion industry leads fashion brands and retailers to create new jobs such as fashion data analysts. The main responsibility of fashion data analysts includes utilizing digital information to predict consumer trends and behavior to maximize profits. According to Fashion United, a global fashion B to B platform (www.fasionunited.com), fashion brands such as Nike, Pandora, Under Armour, and Sweaty Betty have been hiring data analysts who collect, analyze, and define data from the brand's digital channels and present meaningful information to decision makers within the company. In particular, fashion data analysts may have a degree in the STEM fields; but they also need knowledge in fashion merchandising, fashion retailing, and fashion consumer behavior to predict trends and to gain consumer insight better..$^{15} \mathrm{~A}$ good understanding of fashion fundamentals and data analytics should be considered as essential competencies by fashion brands and retailers when they are looking for new talents these days.

\section{Conclusion}

Within the past five years, a small number of fashion programs in higher education have started offering certification courses on optimizing data analytics and AI-based technologies to educate fashion students to think strategically about data-driven decision making and to incorporate data analytics into designing and merchandising strategies. ${ }^{16}$ Data analytics programs now need to be developed in the existing fashion programs in higher education to equip students with the skills and knowledge essential for making data-driven decision using consumer data. The demand for employees with skills and knowledge in fashion data analytics is rapidly glowing as the whole fashion industry has been increasingly valuing the power of data analytics. To implement data analytics in the existing fashion programs, collaboration with technology partners who provide AI-powered data analytics services to fashion brands and retailers is needed. Fashion educators also focus on nurturing students to become digitally curious and adaptable to new technologies. Fashion fundamentals are still essential knowledge; but developing technology 
and data literacy is what is needed for future fashion professionals as the whole fashion industry has become more digitalized.

\section{Acknowledgments}

None.

\section{Funding}

None.

\section{Conflicts of interest}

The authors have no conflicts of interest regarding the publication of this paper.

\section{References}

1. Fox R, Graul M, Peng A, et al. Geek meets chic: Four actions to jumpstart advanced analytics in apparel. Digital/McKinsey: Insights; 2018.

2. Lockwood L. Survey cites dramatic increase in data-driven marketing. WWD; 2013.

3. Roshltsh K. How to localize assortments with data-driven insights. WWD; 2019.

4. What is data analytics? Master's in Data Science; 2020.

5. Turra A. Big data, storytelling and customization were the big trends at Decoded Fashion Milan. WWD; 2015.
6. Cesbron-Lavau E. Think tank: Are you maximizing your data's value? WWD; 2018.

7. Gruzbarg T. How to harness the four dimensions of customer data. WWD; 2019.

8. Zaczklewlcz A. Buried in data? Prescriptive analytics helps fashion retailers sift through the numbers. WWD; 2018.

9. Pastore A. Fit Analytics launches new personalized fit solution, WWD; 2019.

10. Skorupa J. The future of AI-driven merchandising. RIS; 2019.

11. Ma F. Skypad, which captures data trends for luxury fashion, launches in London. WWD; 2020.

12. Doupnik E. Chico's taps first insight for predictive analytics tools. FIRSTINSIGHT; 2017.

13. Young VM. Data analytics firm Custora secures $\$ 17.75 \mathrm{M}$ Series B Raise. WWD; 2018.

14. Olsen L. Data science key to TechStyle fashion group's success. WWD; 2017.

15. Yu A. Fashion careers: What does a fashion data analyst do? Fashion United; 2019.

16. Doupnik E. Fashion institute of technology, first insight partner for data-focused courses. WWD; 2017. 